\title{
Hereditary Orotic Aciduria
}

National Cancer Institute

\section{Source}

National Cancer Institute. Hereditary Orotic Aciduria. NCI Thesaurus. Code C98944.

An extremely rare autosomal recessive inherited disorder caused by mutations in the UMPS gene. It is characterized by deficiency of the activity of the pyrimidine pathway enzyme uridine 5'-monophosphate (UMP) synthase. Clinical manifestations include growth retardation, anemia, and increased excretion of orotic acid in the urine. 